\title{
Network-based RTK Performance for Drone Navigation
}

\author{
Tae-Suk Bae ${ }^{1, *}$ \\ ${ }^{1}$ Sejong University Seoul Korea
}

\begin{abstract}
The Real-time Kinematic (RTK) was predominantly used for the positioning of the ground applications including moving vehicles. Due to the limited baseline lengths, the Network-based RTK (NRTK) was introduced since late-1990s, and successfully applied to the high precision surveying purposes for more than a decade. We conducted the experiments to analyze the performance of the single-baseline RTK (SRTK) as well as the NRTK using one of the operational NRTK systems of Korea, which is the Virtual Reference Stations (VRS) technique. A total of 46 Continuously Operating Reference Stations (CORS) are used for both SRTK and NRTK to secure the redundancy of the baseline lengths. Since the CORS are quite evenly located at an average of 50-60 km, the analysis of error behavior is restricted in terms of seamless variation of baseline length. Therefore, on top of the existing CORS, we simulated the GNSS RINEX data of the rover (that is, the drone position) at a specific location to get the uniform distribution to the reference stations. Furthermore, the rover was generated for different altitude to evaluate the performance of vertical component of the NRTK system. The CORS GNSS data were obtained at $1 \mathrm{~Hz}$ in RINEX format, and the low-cost receiver (ublox) was used for the rover with same interval, single-frequency capability. The correction information from the NRTK server was simultaneously logged for post-processing using the open source software RTKLIB. The percentage of ambiguity resolution was quite reasonable up to $50 \mathrm{~km}$, and drastically drops down as the baseline gets longer. The generated VRS was located with an offset of a few meters in a certain direction, and the horizontal errors also showed a similar pattern. The vertical error behavior due to the tropospheric model deficiency should also be analyzed further.
\end{abstract}

\section{Introduction}

The conventional Global Navigation Satellite System (GNSS) surveying was conducted by either the absolute positioning based on C/A code for low accuracy navigation purpose or the Real-time Kinematic (RTK) technique for surveying and/or geodetic purposes. The RTK has been successfully applied to many applications of precision positioning, especially the real-time applications of moving objects. However, the weakness of RTK is that the accuracy is closely dependent on the distance between the rover and the reference station. This is because the common receiving environment of two receiver positions tends to be spatially decorrelated for longer baseline. Therefore, the accuracy declines beyond a certain limit in the baseline length.

The Network-based RTK (NRTK) was proposed to overcome the drawback of the traditional RTK technique $[1,2,3]$. It was successfully applied to many applications, mostly on the real-time surveying or the moving rover. The accuracy of operational NRTK was compared to that of a single-baseline RTK (SRTK) in terms of baseline lengths. In addition, the characteristics of the positioning accuracy were analyzed as well with respect to the actual RTKs in this study.
The Continuously Operating Reference Station (CORS) network is configured in the southern area of the Korean peninsula. Figure 1 shows the distribution of the CORS network that is being operated by the National Geographic Information Institute (NGII) of Korea. The baseline length is about $60 \mathrm{~km}$ on average to the neighboring CORS. All reference stations are equipped with a geodetic grade GNSS receiver, and the antenna is installed on a pillar that meets the International GNSS Service (IGS) standard specification [4].

The collected data is transferred to the NGII server via Networked Transport of RTCM via Internet Protocol (NTRIP) data stream in real time [5]. The GNSS observations can be downloaded as either daily or hourly observation files at later times. The rover antenna was installed on the rooftop of a building in Seoul which is located in the northwestern part of Korea. The data was logged at 1-second interval $(1 \mathrm{~Hz})$ with an elevation angle cutoff of 15 degrees.

\section{The GNSS reference network}

\footnotetext{
*Corresponding author: author@e-mail.org
} 


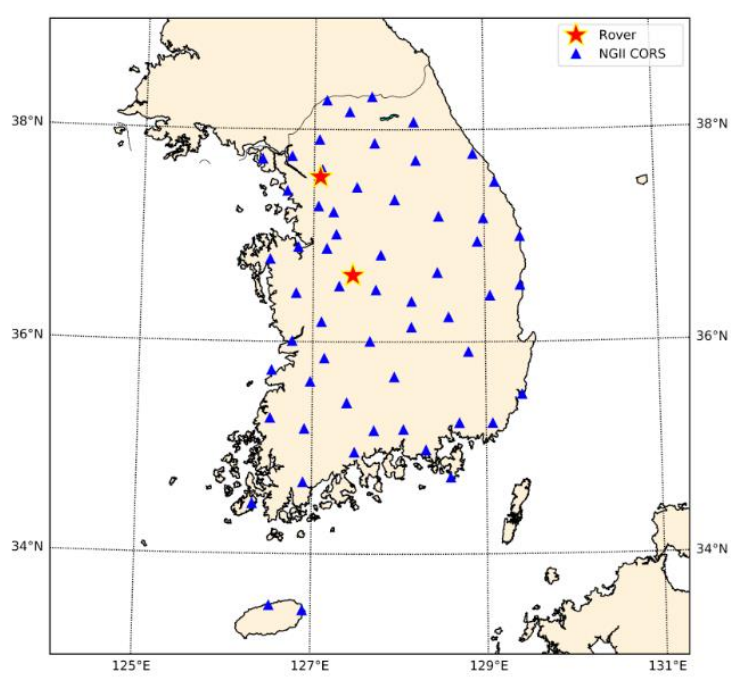

Fig. 1. The configuration of the reference network in Korea.

\section{The Network RTK (NRTK) positioning}

Currently, two NRTK systems are operational by NGII of Korea, that is, Virtual Reference Station (VRS) [6] and Flächen Korrektur Parameter (FKP).

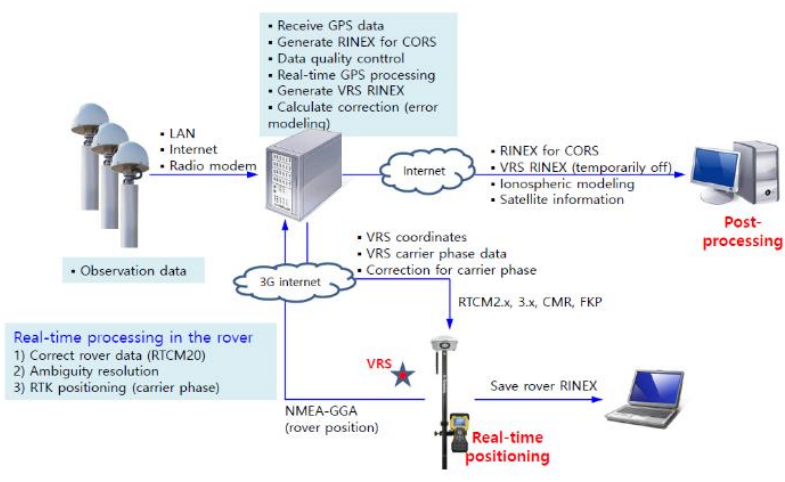

Fig. 2. The system architecture of network-based RTK.

Two systems are designed with a similar concept and provide an equivalent performance in terms of positioning accuracy and ambiguity fixing rates.

Figure 2 shows the system architecture of the NRTK system. All GNSS measurements transferred to the server are converted into RINEX files, and the data quality is instantly evaluated. Once the rover sends its approximate coordinates to the server in an NMEA message, the server generates the virtual reference near the rover position. The correction message in RTCM format (or an equivalently proprietary message such as CMR/CMR+) includes all information for RTK positioning at the rover [7]. The messages are transmitted back and forth through the internet with cellular communication $(3 \mathrm{G} / 4 \mathrm{G})$.

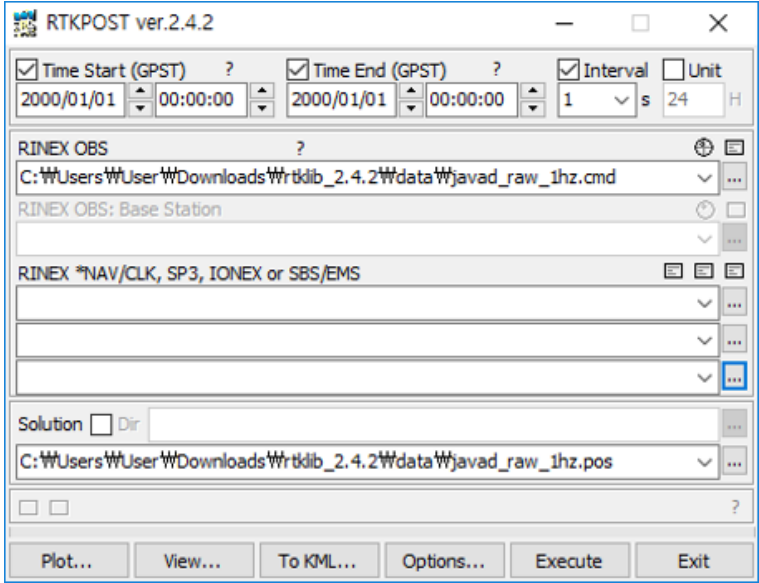

Fig. 3. GNSS positioning software RTKPOST.

The RTK positioning, including the NRTK, was conducted in post-processing mode. The open source software package RTKLIB [8] was used for logging the correction information as well as processing the baselines. Either the RINEX file or the logged correction information was input to the processing software as can be seen in Figure 3. The broadcasting navigation message was used for the satellite orbit and the clock information. The positioning results can be simultaneously stored with the same time interval $(1 \mathrm{~Hz})$. The output can be expressed in global Cartesian coordinates or the local plane at the rover position. Only the ambiguity fixed solutions were considered to calculate the statistics in this study.

The estimated coordinates and the variance-covariance matrix are given in the global Cartesian coordinates system. These need to be transformed into the local frame to analyze the characteristics of the solution. Eq. (1) represents the rotation matrix from the global coordinates system to local (planar) frame. The variance-covariance matrix, $Q$, should also be transformed according to the relationship as given in Eq. (2).

$$
\begin{gathered}
R=\left[\begin{array}{ccc}
-\sin \varphi \cos \lambda & -\sin \varphi \sin \lambda & \cos \varphi \\
-\sin \lambda & \cos \lambda & 0 \\
\cos \varphi \cos \lambda & \cos \varphi \sin \lambda & \sin \varphi
\end{array}\right] \\
Q_{\text {neu }}=R \cdot Q_{X Y Z} \cdot R^{T}
\end{gathered}
$$

where $\varphi$ and $\lambda$ are the geodetic latitude and longitude, respectively.

\section{Experiments of long baseline RTK}

As discussed above, both SRTK and NRTK was accomplished using the package software of RTKLIB (see Table 1) where the processing is done with dualfrequency measurements. Although multi-GNSS signals can be obtained, only the GPS signals were used in this study. The Saastamoinen model was applied for tropospheric delay correction. For the maximum 
redundancy of the analysis, the elevation cutoff angle was set to 15 degrees, and both a Kalman forward filter and backward smoothing were applied for consistent solutions.

Table 1. The specification of the GNSS RTK positioning.

\begin{tabular}{|c|c|}
\hline & Model \\
\hline Receiver/antenna & Geodetic grade \\
\hline Satellites & Dual frequency \\
\hline Frequency & Broadcast message \\
\hline Ephemeris & Saastamoinen model \\
\hline $\begin{array}{c}\text { Tropospheric } \\
\text { model }\end{array}$ & $15^{\circ}$ \\
\hline Elevation cutoff & Forward / backward \\
\hline Filter &
\end{tabular}

Two kinds of experiments were conducted to analyze the effect of the baseline lengths on the positioning accuracy. The SRTK was carried out for the rover in Seoul to all CORS in Korea. In addition, the same procedure was applied for one of CORS to increase the test cases. The NRTK was performed based on VRS that is generated near CORS (same procedure as SRTK).

\section{Test results}

Figure 4 shows the output of SRTK and NRTK positioning. The VRS generated from the server was plotted on the left side, and the horizontal errors from both RTKs are plotted together on the right side. As can be seen in the figure, VRS locations are biased to northeastern direction, and can be classified into two groups.
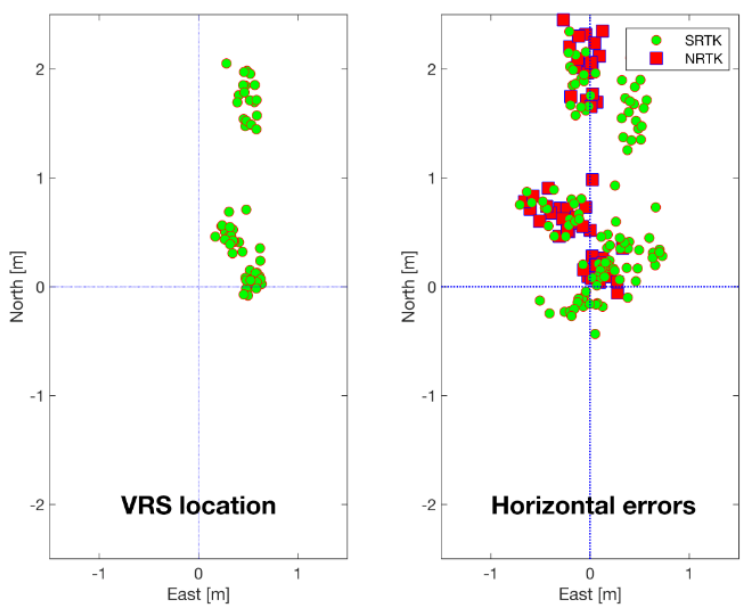

Fig. 4. Error ellipses of RTK solutions.

The average offsets of the VRS are $76 \mathrm{~cm}$ and 45 $\mathrm{cm}$ for the north and east component, respectively. One thing to be noticed is that the error behavior for the NRTK as well as the SRTK solutions is very close to the VRS locations. The accuracy performance of two RTKs is almost comparable in this experiment. This attributes to the fact that most of the RTK processing was conducted for the baselines longer than $50 \mathrm{~km}$. The statistics of the SRTK and NRTK solutions are summarized in Table 2.
The Root Mean Squared Error (RMSE) of NRTK seems slightly worse than that of SRTK.

Table 2. The overall statistics of single-baseline RTK and the Network-based RTK.

\begin{tabular}{|c|c|c|c|c|c|c|}
\hline & \multicolumn{4}{|c|}{ Single-baseline } & \multicolumn{3}{c|}{ Network-based } \\
& $\mathrm{N}$ & $\mathrm{E}$ & $\mathrm{U}$ & $\mathrm{N}$ & \multicolumn{1}{c|}{$\mathrm{E}$} & $\mathrm{U}$ \\
\hline Mean $[\mathrm{m}]$ & 0.7 & 0.1 & 1.9 & 1.0 & -0.1 & 1.8 \\
\hline Std. Dev. $[\mathrm{m}]$ & 0.8 & 0.3 & 1.2 & 0.8 & 0.2 & 1.4 \\
\hline RMSE [m] & 1.1 & 0.3 & 2.3 & 1.3 & 0.2 & 2.3 \\
\hline
\end{tabular}

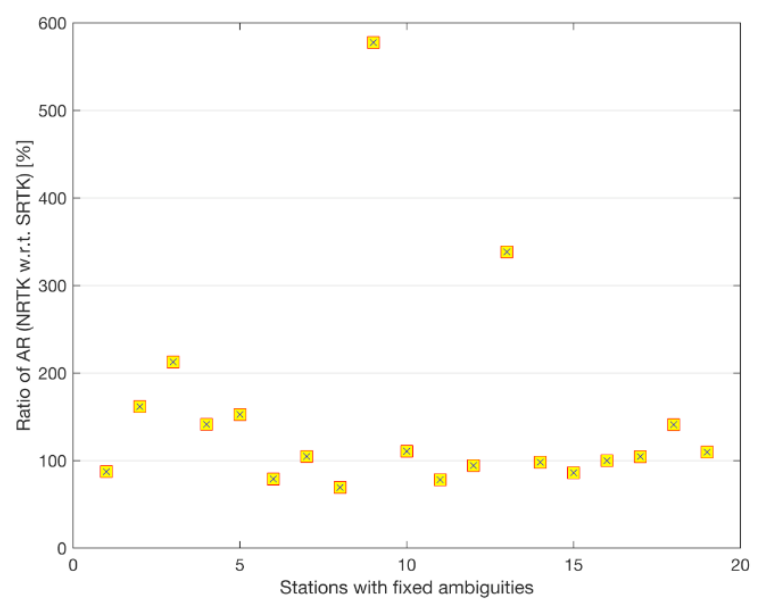

Fig. 5. The ratio of ambiguity resolution for two RTKs.

Figure 5 represents the ratio of ambiguity resolution for two RTK solutions, which is the key success factor. The NRTK shows an improvement in ambiguity resolution (about $150 \%$ on average), although some of the stations show a similar or slightly worse performance. Therefore, the NRTK has an equivalent or better performance in the ambiguity resolution under the same experimental conditions.

As can be seen in Figure 6, most of the baselines are longer than $100 \mathrm{~km}$ since the rover is located in the upper left corner of the (southern) Korean peninsula. The ambiguities are sufficiently resolved for short baselines. However, the success rate rapidly drops down for longer baselines of $50 \mathrm{~km}$. Furthermore, it was almost impossible to resolve the ambiguities over $100 \mathrm{~km}$, except one exceptional station near $100 \mathrm{~km}$. For stations with baselines shorter than $50 \mathrm{~km}$, the NRTK has better performance in resolving the ambiguities.

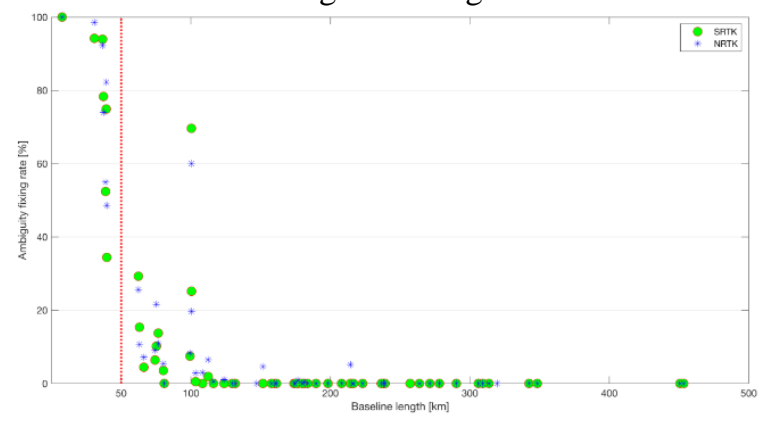

Figure 6: Error ellipses of RTK solutions

\section{Conclusion}


The experiment was conducted to precisely determine the dependency of the baseline lengths of the RTKs. The performance of the SRTK as well as the NRTK generally degrades as the baseline gets longer. The NRTK shows a better performance in terms of success rate of ambiguity resolution. Especially, the ambiguities are reasonably resolved up to $50 \mathrm{~km}$ of baselines while it sharply drops down to the marginal state over $100 \mathrm{~km}$.

The horizontal errors of the RTK solution show that the slope of the north component is larger than that of the east component, which is possibly due to the geometric configuration of the RTK processing. The actual VRS is generated within about $2 \mathrm{~m}$ from each CORS, and the amount of horizontal error corresponds to the bias of VRS locations.

The NRTK positioning assumes the rover to be located within the reference network. Therefore, it needs to be investigated further to apply the NRTK for the extrapolated cases.

This research was supported by the project "Development of soil moisture based on GNSS reflective signals (KMI2018-05710).”

\section{References}

[1] W. Tang, X. Meng, C. Shi, and J. Liu, "Algorithms for sparse network-based RTK GPS positioning and performance assessment," The Journal of Navigation, vol. 66, pp. 335-348 (2013)

[2] U. Vollath, A. Buecherl, H. Landau, C. Pagels, and B. Wagner, "Multi-base RTK positioning using virtual reference stations," Proceedings of ION GPS 2000, Salt Lake City, UT, pp. 123-131 (2000)

[3] G. Wubbena, A. Bagge, and M. Schmitz, "Network-based techniques for RTK applications," GPS JIN 2001 (2001)

[4] M. Kim, Algorithm Development of Integrated Navigation System for Ground Vehicle Using Network-RTK, Sejong University, Master's thesis, 134p (2015)

[5] M. H. Kim and T. S. Bae, "Preliminary analysis of network-RTK for navigation," Journal of the Korean Society of Surveying, Geodesy, Photogrammetry and Cartography, vol. 33, no. 5, pp. 343-351 (2015)

[6] G. R. Hu, H. S. Khoo, P. C. Goh, and C. L. Law, "Development and assessment of GPS virtual reference stations for RTK positioning," Journal of Geodesy, vol. 77, pp. 292-302 (2003)

[7] N. Talbot, G. Lu, T. Allison, and U. Vollath, "Broadcast network RTK-transmission standards and results," Proceedings of the Proceedings of the 15th International Technical Meeting of the Satellite Division of The Institute of Navigation (ION GPS 2002) (2002)

[8] T. Takasu, RTKLIB: An open source program package for GNSS positioning, http://www.rtklib.com (2018)

[9] T. Takasu and A. Yasuda, "Development of the low-cost RTK-GPS receiver with an open source program package RTKLIB," International Symposium on GPS/GNSS (2009) 